\title{
Von Koch and Thue-Morse revisited
}

\author{
J.-P. Allouche \\ CNRS, LRI \\ Bâtiment 490 \\ F-91405 Orsay Cedex (France) \\ allouche@lri.fr
}

\author{
G. Skordev \\ CEVIS, Universität Bremen \\ Universitätsallee 29 \\ D-28359 Bremen (Germany) \\ skordev@cevis.uni-bremen.de
}

\begin{abstract}
We revisit the relation between the von Koch curve and the Thue-Morse sequence given in a recent paper of Ma and Goldener by relating their study to papers written by Coquet and Dekking at the beginning of the $80 \mathrm{~s}$. We also emphasize that more general links between fractal objects and automatic sequences can be found in the literature.
\end{abstract}

\section{Introduction}

The recent paper of Ma and Holdener [27] gives an interesting explicit relation between the von Koch curve and the Thue-Morse sequence. We show here that such a link was already noted in two papers dated 1982-1983, one by Coquet [8] (where the name "von Koch" is not explicitly mentioned, but a construction à la von Koch is given [8, p. 111]), the other by Dekking, see [11, p. 32-05 and p. 32-06]. We explain the similarity of the points of view of these three papers through the approach of Dekking in [9] (where the Thue-Morse sequence does not appear). Actually the fundamental object is the sequence $\left((-1)^{s_{2}(n)} j^{n}\right)_{n \geq 0}$ and its summatory function, where $s_{2}(n)$ is the sum of the binary digits of the integer $n$, hence $\left((-1)^{s_{2}(n)}\right)_{n \geq 0}$ is the \pm 1 Thue-Morse sequence, and $j=e^{2 i \pi / 3}$. The sum $\sum_{n<N}(-1)^{s_{2}(n)} j^{n}$ was explicitly introduced by Coquet in 8 to compute $\sum_{n<N}(-1)^{s_{2}(3 n)}$, and in a more general form by Dekking in [11]. Joining the points of the complex plane that correspond to the sums $\sum_{n<N}(-1)^{s_{2}(n)} j^{n}$ gives a non-renormalized version of the von Koch curve. We end this paper with a quick survey of some general relations between automatic sequences and fractal objects.

We suppose that the reader is familiar with the construction of the von Koch curve (see in particular the original paper of von Koch [26] or the book of Mandelbrot [28]) and with the Thue-Morse sequence (see for example [3]). We will nevertheless recall the definition of the Thue-Morse sequence as well as the definition of morphisms of the free monoid and of automatic sequences: for more details the reader can look at [4] for example. 


\section{A few definitions from combinatorics on words}

In what follows an alphabet is a nonempty finite set. Its elements are called letters. A word on the alphabet $\mathcal{A}$ is a finite string of elements of $\mathcal{A}$. The length of a word is the number of letters composing this word. The concatenation of two words $v=a_{1} a_{2} \cdots a_{i}$ and $w=b_{1} b_{2} \cdots b_{j}$, where the $a$ 's and $b$ 's are letters is noted $v w$ and defined by $v w:=a_{1} a_{2} \cdots a_{i} b_{1} b_{2} \cdots b_{j}$. The free monoid generated by $\mathcal{A}$ is the set of words on $\mathcal{A}$, including the empty word, equipped with the concatenation of words: it is denoted by $\mathcal{A}^{*}$.

Definition 1 Let $\mathcal{A}$ be an alphabet.

A morphism $\varphi$ on $\mathcal{A}$ is a map from $\mathcal{A}^{*}$ to itself that is a homomorphism for the concatenation. (The morphism $\varphi$ is determined by the images $\varphi(a)$ for $a \in \mathcal{A}$.)

A morphism $\varphi$ on $\mathcal{A}$ is called $d$-uniform for some integer $d \geq 1$ (or uniform of length $d$ or uniform if $d$ is clear from the context) if the words $\varphi(a)$ have length $d$ for all $a \in \mathcal{A}$.

Example Let $\mathcal{A}:=\{0,1\}$. Define the 2 -uniform $\varphi$ on $\mathcal{A}$ by $\varphi(0):=01, \varphi(1):=10$. Then, denoting by $\varphi^{k}$ the $k$ th iterate of $\varphi$, we have:

$$
\varphi(0)=01, \quad \varphi^{2}(0)=0110, \quad \varphi^{3}(0)=01101001, \quad \ldots
$$

It is easy to see that the sequence of words $\left(\varphi^{k}(0)\right)_{k}$ simply converges to the infinite sequence

$$
0110100110010110 \ldots
$$

called the Thue-Morse sequence on the alphabet $\{0,1\}$, and that, for $n \geq 0$, the $n$th term of this sequence is equal to the parity of $s_{2}(n)$, the sum of the binary digits of the integer $n$. Replacing 0 by $a$ and 1 by $b$ yields the Thue-Morse sequence on the alphabet $\{a, b\}$ (called the \pm 1 Thue-Morse sequence if $a=1$ and $b=-1$ )

$$
a b b a b a a b b a a b a b b a \ldots
$$

Definition 2 Let $\mathcal{A}$ be an alphabet and $d$ be a positive integer. An infinite sequence $\left(u_{n}\right)_{n \geq 0}$ on $\mathcal{A}$ is called $d$-automatic if there exist an alphabet $\mathcal{B}$, a letter $b \in \mathcal{B}$, a $d$-uniform morphism $\varphi$ on $\mathcal{B}$ and a map $f: \mathcal{B} \rightarrow \mathcal{A}$ such that the sequence of words $\left(\varphi^{k}(b)\right)_{k}$ simply converges to an infinite sequence $\left(v_{n}\right)_{n \geq 0}$ on $\mathcal{B}$, and such that for all $n \geq 0$ we have $u_{n}=f\left(v_{n}\right)$.

\section{The result of $\mathrm{Ma}$ and Holdener}

The authors of [27, p. 202] describe the picture obtained with a "turtle program" (see [1] for example, see also 25]) based on the Thue-Morse sequence. After scaling, the picture converges to the von Koch snowflake. Up to designing a "simplified" curve, the authors prove in fact that the classical iterative construction scheme for the von Koch curve is precisely given by the Thue-Morse sequence in this setting. We begin with a simplified formulation of their result. 
Theorem (Ma, Holdener [27]) Let $\{F, L\}$ be the turtle alphabet where $F$ is move one unit forward and $L$ is rotate by $\pi / 3$. Let $T M_{k}$ be the prefix of length $2^{k}$ of the ThueMorse sequence on the alphabet $\{F, L\}$. Let $W_{n}:=K_{2 n}\left(T M_{4 n}\right)$ be defined as follows: the word $T M_{4 n}$ is written as the concatenation of words from $\left\{T M_{2 n}, \overline{T M}_{2 n}\right\}$, where $\overline{T M}_{2 n}$ is obtained from $T M_{2 n}$ by interchanging the symbols $F$ and $L$; this concatenation is interpreted as a word on the turtle alphabet $\{F, L\}$ thus defining a polygonal line in the plane denoted by $\widetilde{W}_{n}$. Let $S_{n}$ be the scaling factor defined by $S_{n}:=2 /\left(3^{n-2}-1\right)$. Then the sequence of polygonal lines $S_{2 n} \widetilde{W}_{n}$ converges to the von Koch curve.

Proof. This is essentially Theorem 5.0.14 of [27].

One sees that, up to scaling, the polygonal line $\widetilde{W}_{n+1}$ is obtained from $\widetilde{W}_{n}$ in four steps: draw $\widetilde{W}_{n}$, glue a copy of $\widetilde{W}_{n}$ rotated by $-\pi / 3$, glue a copy of $\widetilde{W}_{n}$ rotated by $-\pi / 3+2 \pi / 3=$ $\pi / 3$, and glue a last copy of $\widetilde{W}_{n}$ rotated by $-\pi / 3+2 \pi / 3-\pi / 3=0$. In other words, the polygonal line $\widetilde{W}_{n}$ is transformed into $\widetilde{W}_{n+1}$ exactly as follows, up to scaling: each segment in $\widetilde{W}_{n}$ is interpreted as a complex number $z$ in the coordinates where the origin is an endpoint of this segment and the $x$-axis is parallel to this segment; this complex number is then replaced by the four complex numbers $z,-j z,-j^{2} z, z$ (where $j:=e^{2 i \pi / 3}$ ); the segments corresponding to $z,-j z,-j^{2} z, z$ in the same coordinates are glued consecutively, i.e., the segment we started from is replaced by the segments corresponding to the complex numbers $z, z-j z, z-j z-j^{2} z, z-j z-j^{2} z+z$.

In other words, still ignoring scaling, the curve which is obtained is the geometric representation of the sum $\sum_{0 \leq k<4^{n}} u_{k}$ where the sequence $\left(u_{n}\right)_{n \geq 0}$ is the fixed point beginning in 1 of the morphism defined over the alphabet $\left\{1,-1, j,-j^{2}\right\}$ by

$$
\lambda:\left\{\begin{array}{rlrrrr}
1 & \rightarrow & 1 & -j & -j^{2} & 1 \\
-1 & \rightarrow & -1 & j & j^{2} & -1 \\
j & \rightarrow & j & -j^{2} & -1 & j \\
-j & \rightarrow & -j & j^{2} & 1 & -j \\
j^{2} & \rightarrow & j^{2} & -1 & -j & j^{2} \\
-j^{2} & \rightarrow & -j^{2} & 1 & j & -j^{2}
\end{array}\right.
$$

which easily implies by induction on $n$ that

$$
u_{n}=(-1)^{s_{2}(n)} j^{n}
$$

where $s_{2}(n)$ is the sum of the binary digits of the integer $n$, and where thus the sequence $\left((-1)^{s_{2}(n)}\right)_{n \geq 0}$ is the \pm 1 Thue-Morse sequence. Note that, to recover the usual (bounded) von Koch curve, the appropriate scaling here is $3^{-n}$.

This yields two consequences:

- The occurrence of the Thue-Morse sequence in [27] parallels its occurrence in [8, where Coquet, interested in the behavior of the summatory function $\sum_{k<n}(-1)^{s_{2}(3 k)}$, introduced the sum $\sum_{k<n}(-1)^{s_{2}(k)} j^{k}$. He obtains (page 111) as a by-product the von Koch curve, which he calls "a fractal object built on a classical fractal scheme" (see also [11]). 
- The morphism above is a simplification of the morphism given by Dekking in [9] to construct the von Koch curve. Namely, Dekking considered the morphism defined on the 8-letter alphabet $\left\{s, n, o, w, s^{\prime}, n^{\prime}, o^{\prime}, w^{\prime}\right\}$ by

$\begin{array}{llllll}s & \rightarrow & s & n & o & w \\ w & \rightarrow & s & n & o & w \\ o & \rightarrow & o & w & n^{\prime} & o \\ n & \rightarrow & n & o^{\prime} & s & n \\ s^{\prime} & \rightarrow & s^{\prime} & n^{\prime} & o^{\prime} & w^{\prime} \\ w^{\prime} & \rightarrow & s^{\prime} & n^{\prime} & o^{\prime} & w^{\prime} \\ o^{\prime} & \rightarrow & o^{\prime} & w^{\prime} & n & o^{\prime} \\ n^{\prime} & \rightarrow & n^{\prime} & o & s^{\prime} & n^{\prime}\end{array}$

followed by the map $s \rightarrow 1, w \rightarrow 1, n \rightarrow-j^{2}, o \rightarrow-j, s^{\prime} \rightarrow-1, w^{\prime} \rightarrow-1, n^{\prime} \rightarrow j^{2}$, $o^{\prime} \rightarrow j$ (actually there is a misprint in Dekking's paper for the images of $o$ and $o^{\prime}$ by this map). Dekking's morphism clearly gives the same fixed point beginning with $s$ as the morphism

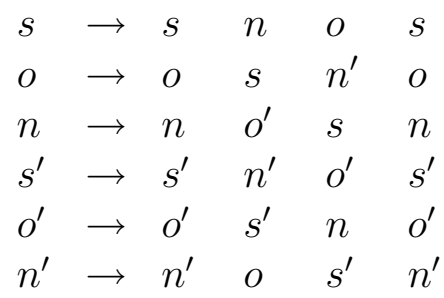

followed by the map $s \rightarrow 1, n \rightarrow-j^{2}, o \rightarrow-j, s^{\prime} \rightarrow-1, n^{\prime} \rightarrow j^{2}, o^{\prime} \rightarrow j$. This last map being a bijection from $\left\{s, n, o, s^{\prime}, n^{\prime}, o^{\prime}\right\}$ to $\left\{1,-1, j,-j, j^{2},-j^{2}\right\}$, we can write this morphism directly on the alphabet $\left\{1,-1, j,-j, j^{2},-j^{2}\right\}$ without changing the final sequence:

$$
\begin{array}{rlrrrr}
1 & \rightarrow & 1 & -j^{2} & -j & 1 \\
-j & \rightarrow & -j & 1 & -j^{2} & -j \\
-j^{2} & \rightarrow & -j^{2} & j & 1 & -j^{2} \\
-1 & \rightarrow & -1 & j^{2} & j & -1 \\
j & \rightarrow & j & -1 & -j^{2} & j \\
j^{2} & \rightarrow & j^{2} & -j & -1 & j^{2}
\end{array}
$$

(this morphism is the morphism $\lambda$ we obtained above up to changing $j$ into $j^{2}$ ).

\section{A generalization}

Let $p$ and $q$ be two integers $\geq 2$. Define $s_{p}(n)$ to be the sum of digits in the $p$-ary expansion of the integer $n$. Define $\eta_{p}:=e^{2 i \pi / p}$ and $\eta_{q}=e^{2 i \pi / q}$. The general sum

$$
Z(N, p, q):=\sum_{0 \leq k<N} \eta_{p}^{s_{p}(k)} \eta_{q}^{k}
$$


was considered by Dekking in [11. In the case where $p=2$, hence $\eta_{p}=-1$ and $s_{p}=s_{2}$, the same procedure as in [8, 9] can be applied, replacing the above morphism of length 4

$$
z \rightarrow z-j z-j^{2} z \quad z
$$

by the morphism of length $Q$

$$
z \quad \rightarrow \quad z \quad(-1)^{s_{2}(1)} \eta_{q} z \quad(-1)^{s_{2}(2)} \eta_{q}^{2} z \quad \cdots \quad(-1)^{s_{2}(Q-1)} \eta_{q}^{Q-1} z
$$

where $Q:=2^{\varphi(q)}$ and $\varphi$ is the Euler function. All details are given in [1], where Dekking gives in particular (see [11, Fig. 1, p. 32-06]) fractal pictures associated with $p=2$ and $q \in\{3,5,7,13\}$ : the case $q=3$, i.e., $Q=4$ and $\eta_{q}=j$, shows of course the von Koch curve.

\section{$5 \quad$ Automatic sequences and fractal objects}

The purpose of this section is to emphasize that there is a general link between automatic sequences and fractal objects. A very first remark is that being a fixed point of a (uniform) morphism means having some sort of self-similarity: since an automatic sequence is infinite, a rescaling process is often necessary to "see" the fractal object. Let us begin with a very simple example. Define the morphism of length 3 on the alphabet $\{0,1\}$ by

$$
0 \rightarrow 000 \quad 1 \rightarrow 101
$$

Its fixed point beginning in 1 is obtained as the limit of the following sequence of words

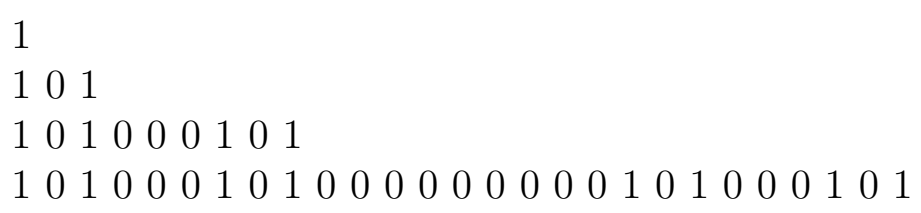

The reader will have immediately noted that, starting with 101, representing 1's by black horizontal segments of length $1 / 3$ and 0 's by white horizontal segments of length $1 / 3$, and "renormalizing" by a factor of $1 / 3$ at each step yields the classical ternary Cantor set. This approach can be generalized to situations where the representation does not necessarily lie on a straight line: see in particular the papers of Golomb [22] and Giles [19, 20, 21] where fixed points of morphisms and automatic sequences are not mentioned but can be found, and the papers of Dekking [9, 10, 11]. In the same direction see the papers of Dekking, Dekking and Mendès France, Deshouillers, Mendès France, and Mendès France and Shallit [12, 13, 15, 29, 30, but also the paper of Salon with an appendix by Shallit [32], where two-dimensional automatic sequences enter the picture, without forgetting the article by Prusinkiewicz and Hammel [31, and the article by Allouche, Allouche and Shallit [2]. We would also like to quote two papers by von Haeseler, Peitgen, Skordev [23, 24] and the references therein, and a paper by Barbé and von Haeseler [5].

Another relation between fractal objects and fixed points of morphisms or automatic sequences $\left(a_{n}\right)_{n \geq 0}$ occurs in the study of the summatory function $\sum_{n<x} a_{n}$, whose asymptotic behavior often involves a logarithmically periodic, continuous and nowhere (or almost 
nowhere) differentiable function: this non-regular behavior corresponds to a geometric object which is fractal. A nice and usually difficult question is to determine the Holder exponents of the non-regular terms in the asymptotic behavior of these summatory functions. While the first such study, due to Delange [14, involves the summatory function of the sequence $\left(s_{2}(n)\right)_{n \geq 0}$, several papers deal with the summatory function of fixed points of morphisms or automatic sequences, or even with more general classes of sequences: see in particular the work of Dumont, and Dumont and Thomas, in particular [16, 17], the thesis of Cateland [6], the paper by Flajolet, Grabner, Kirschenhofer, Prodinger, and Tichy [18], and finally the paper of Tenenbaum [33].

A third direction we want to mention concerns noiselets with in particular an occurrence of the (2-automatic) Shapiro-Rudin sequence, see [7].

What precedes should not hide the specificity of the case addressed here, namely the close relation between the von Koch curve and the Thue-Morse sequence. While the relations explained in this section are quite general, they either link a well-known fractal object to an ad hoc automatic sequence, or a well-known automatic sequence gives rise to some unnamed and not "classical" fractal object. In the case of von Koch and Thue-Morse, the fractal and the sequence were studied quite independently, before a link was found more then eighty years after von Koch's paper and more than seventy years after Thue's paper.

Acknowledgments This paper was written during two stays at Cevis-Mevis of JPA who thanks heartily all the colleagues in Bremen, and in particular H.-O. Peitgen, for their very friendly hospitality. Both authors thank the referee for pertinent and useful comments.

\section{References}

[1] H. Abelson, A. A. diSessa, Turtle geometry. The computer as a medium for exploring mathematics, MIT Press Series in Artificial Intelligence. MIT Press, Cambridge, 1981.

[2] G. Allouche, J.-P. Allouche, J. Shallit, Kolam indiens, dessins sur le sable aux îles Vanuatu, courbe de Sierpinski et morphismes de monoïde, Ann. Inst. Fourier (Grenoble) 56 (2006) 2115-2130.

[3] J.-P. Allouche, J. Shallit, The ubiquitous Prouhet-Thue-Morse sequence, in Sequences and their applications (Singapore, 1998), 1-16, Springer Ser. Discrete Math. Theor. Comput. Sci., Springer, London, 1999.

[4] J.-P. Allouche, J. Shallit, Automatic sequences. Theory, applications, generalizations, Cambridge University Press, Cambridge, 2003.

[5] A. Barbé, F. von Haeseler, Limit sets of automatic sequences. Adv. Math. 175 (2003) 169-196.

[6] E. Cateland, Suites digitales et suites k-régulières, Thèse, Université Bordeaux I, 1992. 
[7] R. Coifman, F. Geshwind, Y. Meyer, Noiselets, Appl. Comput. Harmon. Anal. 10 (2001) $27-44$.

[8] J. Coquet, A summation formula related to the binary digits, Invent. Math. 73 (1983) $107-115$.

[9] F. M. Dekking, Recurrent sets, Adv. in Math. 44 (1982) 78-104.

[10] F. M. Dekking, Replicating superfigures and endomorphisms of free groups, J. Combin. Theory Ser. A 32 (1982) 315-320.

[11] F. M. Dekking, On the distribution of digits in arithmetic sequences, Sém. Théorie des Nombres, Bordeaux, Exposé 32, (1982-1983) 32-01-32-12. Available on line (Göttinger Digitalisierungszentrum) at http://dz-srv1.sub.uni-goettingen.de/sub/digbib/loader?ht=VIEW $\&$ did=D72593

[12] F. M. Dekking, Substitutions, branching processes and fractal sets, in Fractal geometry and analysis (Montreal, PQ, 1989), 99-119, NATO Adv. Sci. Inst. Ser. C Math. Phys. Sci. 346, Kluwer Acad. Publ., Dordrecht, 1991.

[13] F. M. Dekking, M. Mendès France, Uniform distribution modulo one: a geometrical viewpoint, J. Reine Angew. Math. 329 (1981) 143-153.

[14] H. Delange, Sur la fonction sommatoire de la fonction "somme des chiffres", Enseignement Math. 21 (1975) 31-47.

[15] J.-M. Deshouillers, Geometric aspects of Weyl sums, in Elementary and analytic theory of numbers (Warsaw, 1982), 75-82, Banach Center Publ. 17, PWN, Warsaw, 1985.

[16] J.-M. Dumont, Formules sommatoires et systèmes de numération liés aux substitutions, Sém. Théorie des Nombres, Bordeaux, 1987-1988), Exp. No. 39, 39-01-39-12.

[17] J.-M. Dumont, A. Thomas, Systèmes de numération et fonctions fractales relatifs aux substitutions, Theoret. Comput. Sci. 65 (1989) 153-169.

[18] P. Flajolet, P. Grabner, P. Kirschenhofer, H. Prodinger, R. Tichy, Mellin transforms and asymptotics: digital sums, Theoret. Comput. Sci. 123 (1994) 291-314.

[19] J. Giles, Jr, Infinite-level replicating dissections of plane figures, J. Combin. Theory Ser. A 26 (1979) 319-327.

[20] J. Giles, Jr, Construction of replicating superfigures, J. Combin. Theory Ser. A 26 (1979) 328-334.

[21] J. Giles, Jr, Superfigures replicating with polar symmetry, J. Combin. Theory Ser. A 26 (1979) 335-337.

[22] S. W. Golomb, Replicating figures in the plane, Math. Gaz. 48 (1964) 403-412. 
[23] F. von Haeseler, H.-O. Peitgen, G. Skordev, Global analysis of self-similarity features of cellular automata: selected examples, in Chaos, order and patterns: aspects of nonlinearity - the "gran finale" (Como, 1993), Phys. D 86 (1995) 64-80.

[24] F. von Haeseler, H.-O. Peitgen, G. Skordev, On the fractal structure of the rescaled evolution set of Carlitz sequences of polynomials, Discrete Appl. Math. 103 (2000) 89109.

[25] J. Holdener, A. Wagaman, A classification of periodic turtle sequences, Int. J. Math. Math. Sci. 34 (2003) 2193-2201.

[26] H. von Koch, Une méthode géométrique élémentaire pour l'étude de certaines questions de la théorie des courbes planes, Acta Math. 30 (1897) 145-174, Published: 1906. Available on line (Springer) at http://dx.doi.org/10.1007/BF02418570

[27] J. Ma, J. Holdener, When Thue-Morse meets Koch, Fractals 13 (2005) 191-206.

[28] B. B. Mandelbrot, The fractal geometry of nature, W. H. Freeman and Co., San Francisco, 1982.

[29] M. Mendès France, Entropy of curves and uniform distribution, in Topics in classical number theory, Vol. I, II (Budapest, 1981), 1051-1067, Colloq. Math. Soc. János Bolyai 34, North-Holland, Amsterdam, 1984.

[30] M. Mendès France, J.-O. Shallit, Wire bending, J. Combin. Theory, Ser. A 50 (1989) $1-23$.

[31] P. Prusinkiewicz, M. Hammel, Language restricted iterated function systems, Koch constructions and L-systems. In J. C. Hart, ed., New Directions for Fractal Modeling in Computer Graphics, pp. 4-1-4-14, SIGGRAPH '94 Course Notes, July 1994. Available on line at http://citeseer.ist.psu.edu/prusinkiewicz94languagerestricted.html

[32] O. Salon, Suites automatiques à multi-indices, Sém. Théor. Nombres Bordeaux, (19861987), Exposé 4, 4-01-4-27; followed by an appendix by J. Shallit, 4-29A-4-36A.

[33] G. Tenenbaum, Sur la non-dérivabilité de fonctions périodiques associées à certaines formules sommatoires, in The mathematics of Paul Erdös, I, 117-128, Algorithms Combin. 13, Springer, Berlin, 1997. 